\title{
Microalbuminuria in a Cohort of Ambulatory HIV-Positive Nigerians
}

\author{
Komolafe $00^{1^{*}}$, Aderibigbe A $^{1}$, Olanrewaju TO ${ }^{1}$, Chijioke ${ }^{1}{ }^{1}$, Salami AK ${ }^{2}$ and Rafiu MO ${ }^{1}$ \\ ${ }^{1}$ Renal Care Centre, University of Ilorin Teaching Hospital, Ilorin, Nigeria \\ ${ }^{2}$ Department of Medicine, University of Ilorin Teaching Hospital, Ilorin, Nigeria
}

*Corresponding author: Opeyemi O. Komolafe, Dialysis Unit, Garki Hospital, PMB 656, Area 7 Garki, Abuja, FCT 900001, Nigeria, Tel: +234-803 668 8214; E-mail: el_komo@yahoo.com

Rec date: Jun 20, 2014, Acc date: Jul 21, 2014, Pub date: Jul 26, 2014

Copyright: (C) 2014 Komolafe O, et al. This is an open-access article distributed under the terms of the Creative Commons Attribution License, which permits unrestricted use, distribution, and reproduction in any medium, provided the original author and source are credited.

\begin{abstract}
Quantification of urine albumin is an established method of screening, diagnosis and monitoring the progression of kidney disease. Renal disease is a frequent accompaniment of Human Immunodeficiency Virus (HIV) infection. Limited data exists with regard to the epidemiology of microalbuminuria in indigenous African HIV patients. To study this issue further, we evaluated 90 antiretroviral-naïve HIV seropositive adults and compared them with an equivalent number of age and sex matched HIV seronegative controls. Individuals with known confounders of urine albumin were excluded from the study. We found microalbuminuria in $35.6 \%$ of HIV cases as against $13.3 \%$ in the seronegative group $(p=0.001)$. In addition, there was a positive correlation between cluster of differentiation 4 (CD4) cell count and microalbuminuria $(p=0.001)$. Similarly, decreasing estimated glomerular filtration rate (eGFR) correlated with the finding of microalbuminuria. Our findings suggest that microalbuminuria is a frequent finding in adult anti-retroviral naïve seropositive Nigerians particularly in those with lower CD4 cell counts and lower eGFR.
\end{abstract}

Keywords: Microalbuminuria; HIV; Kidney disease; Africans; CD4; eGFR

\section{Introduction}

Microalbuminuria is widely accepted as a measurable marker of early kidney damage [1] and its utility in the evaluation of HIV-related renal disease has been a focus of recent interest [2-4].

Renal disease was first reported in HIV-1 seropositive individuals by Rao and colleagues in 1984 [5], since then various studies have highlighted the diverse manifestations of renal disease in HIV seropositive patients [6-8]. The spectrum of renal disease in HIV infection encompasses microalbuminuria, through proteinuria to EndStage Renal Disease (ESRD) and it appears that the virus exerts its deleterious effect on the kidney via a direct cytopathic effect in the presence of an enabling cytokine milieu among genetically susceptible individuals [9].

Africa bears a disproportionate share of the global HIV burden [10], it can therefore be expected that the prevalence of HIV-related renal disease would be highest in Sub-Saharan Africa where the prevalence of HIV is highest. Furthermore, persons of African origin appear to be more predisposed [11] to the renal manifestations of HIV and when these occur, progression to ESRD is usually more rapid and overall prognosis poorer [12].

Prior to the introduction of highly active antiretroviral therapy (HAART), opportunistic infections accounted for most of the morbidity and mortality among patients with the acquired immune deficiency syndrome (AIDS) [13] Currently, although the incidence of kidney disease has decreased [14], in part due to the advent of HAART, its prevalence is on the rise due to aging and improved life expectancy among HIV-infected patients [13].

For every patient that develops ESRD, there are far-reaching social and economic implications. With this in mind, early risk detection is crucial, so that appropriate treatment can be instituted before extensive damage occurs particularly, in resource-poor settings where treatment access is limited.

Early detection of renal disease in HIV patients using microalbuminuria has not been extensively studied in indigenous African populations [15]. In addition, bench-marks for best practices to limit the renal impact of HIV have not been defined. To address this problem, this study examined the prevalence and correlates of microalbuminuria in Nigerians with HIV infection.

\section{Results}

The study population comprised 90 ART-naïve HIV seropositive cases and 90 age and sex-matched HIV seronegative control subjects who met the criteria for enrollment.

\section{Age distribution}

The age range of the cases was between 18 to 60 years, while the controls were aged between 20 to 58yrs. The mean age \pm standard deviation of the cases was $37.20 \pm 8.79$ years. This was comparable to the mean age \pm standard deviation of the controls which was $37.88 \pm$ 8.68. The difference was not statistically significant $(\mathrm{P}=0.603)$.

\section{Pattern of CD4 cell count among cases}

The CD4 cell count in the case group ranged between 4 cells/ $\mathrm{mm}^{3}$ and 1226 cells $/ \mathrm{mm}^{3}$ with a median value of 371.50 cells $/ \mathrm{mm}^{3}, 29$ (32.2\%) subjects had CD4 cell counts $>500$ cells $/ \mathrm{mm}^{3}, 34(37.8 \%)$ subjects had CD 4 cell counts between 200 and 499 cells $/ \mathrm{mm}^{3}$ while 27 (30.0\%) subjects had CD 4 cell counts $<200$ cells $/ \mathrm{mm}^{3}$. 


\section{Prevalence of microalbuminuria among CD4 groups}

$17(63 \%)$ of 27 patients with CD4 cell counts less than 200 cells $/ \mathrm{mm}^{3}$ had microalbuminuria, 10 (29.4\%) of 34 patients with CD4 cell counts between 200 and 499 cells $/ \mathrm{mm}^{3}$ had microalbuminuria while 5 (17.24\%) of 29 patients with CD4 cell counts greater than or equal to 500 cells/mm3 had microalbuminuria.

\section{Pattern of estimated glomerular filtration rate (eGFR) among cases}

The mean eGFR \pm standard deviation of the cases was $105.73 \pm$ $30.26 \mathrm{mls} / \mathrm{min} / 1.73 \mathrm{~m}^{2}$. $64(71.1 \%)$ subjects had an eGFR of $\geq 90$ $\mathrm{mls} / \mathrm{min} / 1.73 \mathrm{~m}^{2}, 23(25.6 \%)$ had an eGFR of between $60-89.9$ $\mathrm{mls} / \mathrm{min} / 1.73 \mathrm{~m}^{2}, 2$ (2.2\%) subjects had an eGFR of between $30-59.9$ $\mathrm{mls} / \mathrm{min} / 1.73 \mathrm{~m}^{2}$ while 1 (1.1\%) subject had an eGFR of $\leq 14.9$ $\mathrm{mls} / \mathrm{min} / 1.73 \mathrm{~m}^{2}$.

\section{Prevalence of microalbuminuria among eGFR groups}

$17(26.6 \%)$ of 64 patients with eGFR $\geq 90 \mathrm{mls} / \mathrm{min} / 1.73 \mathrm{~m}^{2}$ had microalbuminuria while $12(52.2 \%)$ of 23 patients with eGFR between $60-89.9 \mathrm{mls} / \mathrm{min} / \mathrm{m}^{2}$ had microalbuminuria. Both of the 2 patients with eGFR between $30-59.9 \mathrm{mls} / \mathrm{min} / \mathrm{m}^{2}$ had microalbuminuria and the only patient with eGFR $\leq 14.9 \mathrm{mls} / \mathrm{min} / \mathrm{m}^{2}$ had microalbuminuria.

\section{Prevalence of microalbuminuria}

A total of 58 (64.4\%) individuals in the case group had normoalbuminuria while microalbuminuria was found in the remaining $32(35.6 \%)$ subjects. In the control group, $78(86.7 \%)$ subjects had normoalbuminuria and microalbuminuria was found in $12(13.3 \%)$ members of the control group. The difference in the prevalence between the 2 groups was statistically significant $\left(\mathrm{X}^{2}\right.$ $=12.032 ; \mathrm{df}=1 ;$ P value $=0.001)$.

\section{Correlates of microalbuminuria}

There was a statistically significant positive correlation between CD4 cell count and microalbuminuria ( $\mathrm{Rs}=0.347$; $\mathrm{P}$ value $=0.001)$. In like manner, a statistically significant positive correlation existed between decreasing eGFR and the detection of microalbuminuria (Rs= 0.310; P Value $=0.003$ ).

\section{Discussion}

The primary objective of this study was to define the prevalence and predictors of microalbuminuria in anti-retroviral naïve adult HIV seropositive Nigerians seen at the University of Ilorin Teaching Hospital, Ilorin, Nigeria, to this end, 90 ART-naive HIV seropositive cases and 90 age and sex-matched control subjects who met the enrolment criteria were studied.

This study has 2 key findings: First, 35.6\% of HIV seropositive cases in the study had microalbuminuria. Second, the finding of microalbuminuria correlated with lower CD4 counts and lower eGFR. Several studies have documented the prevalence of microalbuminuria among diabetics, hypertensives and in members of the general population. In contrast, relatively few studies have examined the prevalence of microalbuminuria in HIV seropositive patients. As a result, there is only limited data available on the epidemiology of microalbuminuria in HIV patients. This study demonstrates a high prevalence of microalbuminuria in the studied cohort which is in agreement with findings of previous studies which show a higher prevalence of microalbuminuria in HIV positive persons compared with their HIV negative counterparts $[2,3,4,16,17]$.

Microalbuminuria appears to be a common finding in HIV seropositive patients. In an earlier study of patients with AIDS, microalbuminuria was found in $19.4 \%$ of patients [16]. In another study from Norway, Baekken et al. found a microalbuminuria prevalence of $8.7 \%$ in an HIV-infected cohort [2], this figure was three to five times higher than in the seronegative control group. In the US, Szczech and colleagues found microalbuminuria in $11 \%$ of HIVpositive persons and only $2 \%$ of the control participants [3]. In South Africa, Han et al. [4] evaluated 615 ART-naïve HIV-infected patients between 2002 and 2004. Microalbuminuria was screened for in 90 of the 615 patients, and was found in $36 \%$ of them. Kwaifa and Bosan [17] in Zaria, Nigeria in their study of a cohort of 400 HIV-infected adults attending an HIV clinic in North-Western Nigeria, detected microalbuminuria in $33.1 \%$ of the study participants.

The differences in the prevalence recorded in the above studies may relate to ethnic differences in the study population. Studies that largely involved HIV positive Caucasian subjects seemed to observe a lower prevalence of microalbuminuria compared with studies among patients of Afro-Caribbean extraction. It can be expected that the prevalence of HIV-related renal disease would be higher in persons of African origin, a racial group known to have a high prevalence of HIVAN and other renal diseases $[11,18,19]$.

The finding of a microalbuminuria prevalence of $35.6 \%$ among HIV positive subjects in this study is in tandem with the $36 \%$ prevalence reported in Han's South African study and the $33.1 \%$ prevalence reported from Zaria by Kwaifa. The ethnic profile of the participants in this study closely mirrors that of both Kwaifa and Han's study population and this may in part explain the similarity of the findings.

Less than one third of studied subjects had advanced HIV infection with CD4 cell counts $<200$ cells $/ \mathrm{mm}^{3}$ and therefore, the bulk of respondents were relatively healthy. According to published WHO criteria [20] for staging AIDS, at CD4 cell counts of $<200$ cells $/ \mathrm{mm}^{3}$, the protean multi-systemic manifestations of HIV become more clinically evident. The implication of this is that as CD4 cell count drops, renal manifestations, including microalbuminuria are likely to occur more frequently as various studies have shown $[2,3,4,16,17]$.

It could reasonably be expected that the reported incidence and magnitude of microalbuminuria in this study would be lower than if a higher proportion of cases with CD4 cell counts $<200$ cells $/ \mathrm{mm}^{3}$ were recruited. To further highlight this point, this study showed that over 1 in 2 of subjects with CD4 cell counts less than 200 cells $/ \mathrm{mm}^{3}$ had microalbuminuria in comparison to 1 in 3 in the $200-499 \mathrm{cells} / \mathrm{mm}^{3}$ group and nearly 1 in 5 in the $\geq 500$ cells $/ \mathrm{mm}^{3}$ group. The observation of a significant correlation between $\mathrm{CD} 4$ cell count and microalbuminuria in this study is in agreement with the findings of previous studies $[2,3,4,16,17]$.

Statistically significant differences were observed regarding CD4 cell counts and urine albumin levels between HIV seropositive subjects with microalbuminuria and those with normoalbuminuria. Subjects with microalbuminuria tended to have lower CD4 cell counts than those with normoalbuminuria. In like manner, subjects with microalbuminuria had higher mean urine albumin levels than their HIV seronegative counterparts. Based on these findings, it appears likely that in HIV patients, as CD4 count decreases, microalbuminuria becomes a more frequent finding. 
The finding in this study of lower mean CD4 counts in HIV patients with microalbuminuria may reflect the contribution of increased HIV activity in subjects with lower CD4 counts to the phenomenon of abnormal protein trafficking across the slit diaphragm and glomerular capillary basement membrane.

However, the finding (though less frequent) of microalbuminuria in individual patients with relatively high CD4 counts in this study does not support this hypothesis and indeed raises the possibility that other mechanisms may be involved in the pathogenesis of microalbuminuria in HIV infection.

The Kidney Disease Outcomes Quality Initiative (K/DOQI) clinical practice guidelines on the classification and staging of chronic kidney disease was used to stratify respondents into 5 stages from stage 1 through 5 based on the eGFR [21].

Intuitively, decreasing eGFR would be expected to be associated with microalbuminuria, given that microalbuminuria is an established marker of renal disease [1]. The bulk of HIV-seropositive subjects had an eGFR of $\geq 90 \mathrm{mls} / \mathrm{min} / 1.73 \mathrm{~m}^{2}$ and considering that in this eGFR group, $26.6 \%$ had microalbuminuria, this implies that at the very least, $26.6 \%$ of this group had stage $1 \mathrm{CKD}$, this percentage could actually be higher bearing in mind that an exhaustive search for microscopic or imaging evidence was not undertaken as this was not part of the study objectives or protocol (In patients with stage 1 CKD, although eGFR remains apparently normal; structural or functional evidence of kidney damage may exist.) [21] The prevalence of CKD stage 2,3 and 5 in the study population were $25.6 \%, 2.2 \%$ and $1.1 \%$ respectively.

No patient with stage 4 CKD was found in this study. Therefore, HIV positive subjects in this study had a combined CKD prevalence of $55.5 \%$. This figure was similar to that observed in a Ugandan study [22] that showed a CKD prevalence of $48.5 \%$ in a cohort of HIV seropositive persons. These CKD prevalence figures among HIV positive subjects are disproportionately higher when compared to results from a study in Kinshasa, Congo [23] that showed a CKD prevalence of $12.4 \%$ in the general population. This disparity may be explained in part by the fact that this study and the Ugandan study were hospital-based surveys in known HIV seropositive patients in contrast to the Kinshasa study which was carried out among the general population.

Over $50 \%$ of HIV positive subjects in this study had some degree of $\mathrm{CKD}$ and this further highlights the need for targeted screening for chronic kidney disease among selected high-risk groups. In recognition of the burden of renal disease among HIV-infected persons, it seems likely that screening of HIV-infected individuals could result in significant savings in terms of health for the patients and costs for the nation.

Although it was not a primary objective of the study, we also found differences among certain clinical and laboratory indices, between case and control groups pertaining to body mass index (BMI), systolic blood pressure (SBP), diastolic blood pressure (DBP), packed cell volume(PCV), fasting blood glucose(FBG )and Urine albumin levels.

The observation of lower mean BMI in the case group is not surprising given that soft tissue wasting is a recognized clinical finding in HIV/AIDS [24].

The lower mean SBP and DBP observed among the cases may reflect at least in part, the myriad of fluid and electrolyte disturbances that have been described in HIV positive subjects of which hyponatremia is the most common [7].
Hyponatremia in the setting of HIV/AIDS could result from diverse physiological alterations including excessive salt loss from the gastrointestinal tract or skin, renal salt wasting from tubulointerstitial disease, syndrome of inappropriate anti-diuretic hormone secretion from opportunistic infections of the lungs or central nervous system, adrenal insufficiency as well as administration of hypotonic intravenous solutions [7].

Furthermore, although renal biopsies were not performed in this study, the possibility exists that some of the studied subjects had subclinical HIVAN of which low to normal blood pressure is a recognized clinical feature [25].

Again, the mean PCV was lower in the case group, normocytic, normochromic anemia is a frequent accompaniment of HIV infection, and indeed HIV-infected patients tend to have more significant anaemia than the general chronic kidney disease population [26].

Possible explanations for this phenomenon includes direct and indirect effects of HIV inhibition on red blood cell precursors, increased peripheral destruction of red blood cells and concomitant infection with parvovirus resulting in pure red cell aplasia [26].

Surprisingly however, HIV positive individuals as a group had overall lower FBG levels than their HIV negative counterparts. Despite the finding of higher overall FBG levels in the HIV negative group, no single participant in either case or control groups had FBG levels in the diabetic range; as diabetes mellitus was considered an exclusion criterion for recruitment into the study. The finding of higher overall FBG levels in HIV-seronegative participants is unexpected given the associations that have been made between HIV infection and insulin resistance in previous studies [27].

Of all socio-demographic characteristics studied, only gender appeared to have any relationship to microalbuminuria among HIV positive subjects. This finding may reflect the observation in this study that a significant proportion of HIV seropositive males had microalbuminuria when compared with their female counterparts. Among the $32 \mathrm{HIV}$ seropositive individuals with microalbuminuria studied, $16(50 \%)$ of them were males, despite the fact that males were outnumbered by females in the study population by a ratio of nearly 2 to 1 . In relative terms therefore, a higher proportion of males in this study had microalbuminuria and the effect of male gender on microalbuminuria was statistically significant. The observed clustering of microalbuminuria among male HIV patients in this study mirrors the findings of other studies that suggest that male gender confers a higher risk for microalbuminuria [28,29]. Male HIV seropositive subjects in this study also had higher mean urine albumin levels than their female counterparts although this difference did not reach statistical significance.

This study has several limitations, As a result of the highly restrictive study eligibility requirements, most of the study participants were stable outpatients and this may have resulted in a relatively healthy cohort effect, which would underestimate the actual prevalence of microalbuminuria.

The cross-sectional design of the study precluded sequential measurement of microalbuminuria, which should ideally be done on at least 3 occasions within a period of 3-6 months [30].

Viral load was not measured because of the absence of facilities for measurement. Bearing in mind that HIV viral load may be a more direct indicator of HIV activity and viral burden than CD4 cell count. 
Despite its shortcomings, data from this study demonstrates thatthere is a high prevalence of microalbuminuria amongst ARTnaïve HIV seropositive adults in Nigeria.

This finding reinforces the observations of other studies that show a relatively higher prevalence of microalbuminuria among blacks with HIV infection.

Decreasing CD4 cell count correlated positively with the finding of microalbuminuria among HIV seropositive study participants suggesting that immune status may influence the development of microalbuminuria.

Decreasing eGFR was also associated with microalbuminuria among HIV seropositive study participants.

In conclusion, taken together, the results of this study may have implications for future studies and perhaps risk stratification in clinical practice. Specifically, we recommend that a screening protocol for microalbuminuria should be established as part of the initial evaluation of HIV seropositive individuals who are at increased risk of developing CKD [31] as this may afford the earliest opportunity for detection.

In those confirmed to have microalbuminuria, earlier interventions with disease-specific therapy, and referral to a nephrologist, is likely to retard or even halt the progression of microalbuminuria, leading to better overall outcomes.

Finally, this study further emphasizes the need for longitudinal research in larger cohorts in order to investigate more conclusively the association between HIV infection and microalbuminuria as well as to elucidate with greater clarity the natural history, significance and sequelae of microalbuminuria in HIV infection.

\section{Materials and Methods}

\section{Study location}

The study was carried out at the University Of Ilorin Teaching Hospital (UITH), Ilorin, a 500 bed facility located in North Central Nigeria. The hospital runs an HIV clinic that is supported by the Institute of Human Virology, Nigeria (IHVN) a non-governmental organization, under the auspices of the ACTION (AIDS Care and Treatment in Nigeria) project. The clinic offers counselling services, screening, laboratory tests and treatment free of charge to the patients. The activities of the clinic are funded by IHVN and its partner organizations.

\section{Study Design and Population}

The study was a cross-sectional, case-control study that was carried out between March and October 2010. The Ethics and Research Committee of the UITH approved the study in accordance with the Declaration of Helsinki principles (as revised in 1983). After approval of the protocol by the institution review board, signed informed consent forms were obtained from each study participant. Stringent inclusion criteria were applied to the prospective study participants. The careful selection of patients for inclusion in the study was necessary to avoid interference by known confounders of urine albumin excretion. All consenting anti-retroviral naïve adult HIV seropositive patients aged between 18 and 60 years of age seen at the study location were consecutively recruited into the study.Patients unwilling to give consent for the study were excluded. All study participants had a dipstick urine analysis prior to enrollment in the study and only subjects with negative protein on dipstick testing were included. Furthermore, patients who had conditions that could increase the urine albumin excretion and thus confound the measurement of urine albumin were excluded. Thus, patients with a previous or recent history of kidney disease, those with overt symptoms or signs attributable to kidney disease, bed-bound individuals or those sufficiently ill as to require assistance with usual activities of daily living, acutely ill patients (from any cause), patients with systemic hypertension, diabetes mellitus or sickle cell disease. In addition, patients diagnosed with or being evaluated for cancer, those with symptoms suggestive of urinary tract infection, rhabdomyolysis or intravascular hemolysis were excluded. Finally, subjects with cardiac failure or fever were excluded as were pregnant, menstruating or who had an abnormal vaginal discharge.

Exclusion criteria for the study subjects were also applied to the control participants. The control subjects who were HIV negative were screened after appropriate pre-test counselling and consisted of individuals who visited the hospital for pre-employment medical screening and routine medicals.

A total of 103 HIV seropositive individuals who were seen at the study location between March 2010 and October 2010 were consecutively recruited. I3 patients were however excluded in the course of the study because of; subsequent confirmation of diabetes $(n=2)$ drop out after the initial visit $(n=3)$, visibly contaminated urine samples $(n=3)$ and incomplete data $(n=5)$. Finally, 90 patients who had complete data and fulfilled all inclusion criteria were studied and these were sex and age-matched (within 5yrs) to 90 control subjects.

\section{Variables and Data Sources}

All participants completed a detailed structured questionnaire from which we obtained basic demographic and clinical information. Participants underwent a structured health history and a focused physical examination to ensure compliance with the set criteria. Blood pressure (BP) and BMI were measured in a standardized manner. The study participants also provided blood and urine samples for biochemical analysis. The following laboratory parameters were assessed: FBG, Serum Creatinine, CBC, CD4 cell count and urine albumin. Creatinine was analysed using the standard colorimetric Jaffe kinetic reaction method. Samples for fasting blood glucose estimation were analysed using the glucose oxidase method. Blood for complete blood count was analyzed using an automated analyzer (Sysmex 2100, GMI inc, USA) while blood for CD4 cell count was analysed using an automated flow cytometry machine $\left(\mathrm{CyFlow}^{\circ}\right.$ Counter GMBH, Germany). Briefly, Urine microalbumin measurement was performed using the HemoCue Urine Albumin analyzer (HemoCue $A B$, Angelholm, Sweden) which employs an immunoturbidometric technique in which a specific rabbit anti human albumin polyclonal antibody forms an agglutinin with albumin in the urine sample and the turbidity of the agglutinate is measured photometrically at $610 \mathrm{~nm}$ within 90 seconds. Microcuvettes for the Hemocue Urine Albumin analyzer were stored in a refridgerator at $2-8^{\circ} \mathrm{C}$ according to the manufacturer's recommendation. When possible, the first morning urine sample was collected in plain bottles for analysis and in cases where the patient had already voided, spot urine samples were utilized. All the clinic consultations and sample collections took place in the morning and urine samples were analyzed within an hour of collection. Estimated Glomerular Filtration Rate (eGFR) was 
calculated using the 4-variable Modification of Diet in Renal Disease (MDRD) Study Equation [32].

The 4-variable MDRD formula has been validated in adult Nigerians with CKD [33]. Values were derived by entering data on age, sex, race and serum creatinine into a software programme for GFR calculation (Roche Pharmaceuticals, 2005).

\section{Operational Definitions}

The definitions stated here are with reference to hypertension, microalbuminuria and stages of CKD as used in this study.

Microalbuminuria was defined as a measured urine albumin level of $\geq 30 \mathrm{mg} / \mathrm{l}$.

CKD was staged according to the the Kidney disease outcomes quality initiative (K/DOQI) clinical practice guidelines [32] on the classification and staging of CKD which is an eGFR based classification:

Stage 1- eGFR $\geq 90 \mathrm{mls} / \mathrm{min} / 1.73 \mathrm{~m}^{2}$ with some evidence of kidney damage reflected by microalbuminuria, protenuria, hematuria, histologic or imaging abnormalities.

Stage 2- eGFR between $60-89 \mathrm{mls} / \mathrm{min} / 1.73 \mathrm{~m}^{2}$

Stage 3- eGFR between $30-59 \mathrm{mls} / \mathrm{min} / 1.73 \mathrm{~m}^{2}$

Stage 4- eGFR between $15-29 \mathrm{mls} / \mathrm{min} / 1.73 \mathrm{~m}^{2}$

Stage 5- eGFR $<15 \mathrm{mls} / \mathrm{min} / 1.73 \mathrm{~m}^{2}$

Hypertension was defined as a blood pressure $>140 / 90 \mathrm{mmHg}$ or use of anti-hypertensive agents.

\section{Statistical Analysis}

The frequencies of normally distributed numerical variables were expressed as mean \pm standard deviation while non-parametric numerical variables were expressed as median values. The statistical significance of variables was determined as follows; Pearson's Chisquare test was used to compare proportions of categorical variables, Student's t-test was used to compare means of parametric continuous variables, while Mann-Whitney $U$ test was used to compare continuous variables that were not normally distributed. Spearman's correlation method was used to determine the correlates of microalbuminuria. All $\mathrm{p}$ values are two-tailed and the significance threshold was set at $<0.05$. Data was analyzed using the statistical package for social sciences (SPSS) software, version 16.0 (Chicago, IL, USA).

\section{Contributions}

AA, TOO \& OOK developed the study concept and design, AC \& AKS provided clinical advice, OOK collected the data, MOR \& OOK analysed the data. OOK drafted the manuscript. All authors critically reviewed and approved the manuscript.

\section{Acknowledgments}

All study participants who graciously consented to participate in this research.

\section{References}

1. Rowe DJ, Dawnay A, Watts GF (1990) Microalbuminuria in diabetes mellitus: review and recommendations for the measurement of albumin in urine. Ann Clin Biochem 27 : 297-312.

2. Baekken M, Os I, Sandvik L, Oektedalen O (2008) Microalbuminuria associated with indicators of inflammatory activity in an HIV-positive population. Nephrol Dial Transplant 23: 3130-3137.

3. Szczech LA, Grunfeld C, Scherzer R, Canchola JA, van der Horst C, et al. (2007) Microalbuminuria in HIV infection. AIDS 21: 1003-1009.

4. Han TM, Naicker S, Ramdial PK, Assounga AG (2006) A cross-sectional study of HIV-seropositive patients with varying degrees of proteinuria in South Africa. Kidney Int 69: 2243-2250.

5. Rao TK, Filippone EJ, Nicastri AD, Landesman SH, Frank E, et al. (1984) Associated focal and segmental glomerulosclerosis in the acquired immunodeficiency syndrome. N Engl J Med 310: 669-673.

6. Szczech LA, Gupta SK, Habash R, Guasch A, Kalayjian R, et al. (2004) The clinical epidemiology and course of the spectrum of renal diseases associated with HIV infection. Kidney Int 66: 1145-1152.

7. Peter SA (1991) Electrolyte disorders and renal dysfunction in acquired immunodeficiency syndrome patients. J Natl Med Assoc 83: 889-891.

8. Valeri A, Neusy AJ (1991) Acute and chronic renal disease in hospitalized AIDS patients. Clin Nephrol 35: 110-118.

9. Weiner NJ, Goodman JW, Kimmel PL (2003) The HIV-associated renal diseases: current insight into pathogenesis and treatment. Kidney Int 63: 1618-1631.

10. UNAIDS: Report on the Global HIV/AIDS Epidemic 2008: Executive Summary. Mexico City, Mexico, UNAIDS, 2008.

11. Kopp JB, Winkler C (2003) HIV-associated nephropathy in African Americans. Kidney Int Suppl : S43-49.

12. Lucas GM, Lau B, Atta MG, Fine DM, Keruly J, et al. (2008) Chronic kidney disease incidence, and progression to end-stage renal disease, in HIV-infected individuals: a tale of two races. J Infect Dis 197: 1548-1557.

13. Lima VD, Hogg RS, Harrigan PR, Moore D, Yip B, et al. (2007) Continued improvement in survival among HIV-infected individuals with newer forms of highly active antiretroviral therapy. AIDS 21: 685-692.

14. Lucas GM, Eustace JA, Sozio S, Mentari EK, Appiah KA, et al. (2004) Highly active antiretroviral therapy and the incidence of HIV-1associated nephropathy: a 12-year cohort study. AIDS 18: 541-546.

15. Cohen SD, Kimmel PL (2007) HIV-associated renal diseases in Africa a desperate need for additional study. Nephrol Dial Transplant 22: 2116-2119.

16. Luke DR, Sarnoski TP, Dennis S (1992) Incidence of microalbuminuria in ambulatory patients with acquired immunodeficiency syndrome. Clin Nephrol 38: 69-74.

17. Kwaifa SI, Bosan IB (2008) Chronic Kidney Disease in HIV-infected participants in North-Western Nigeria. Trop J Nephrol 3: 23-28.

18. US Renal Data System (2007) USRDS 2007 Annual Data Report. Atlas of Chronic Kidney Disease and End-Stage Renal Disease in the United States. Bethesda, MD: National Institutes of Health, National Institute of Diabetes and Digestive and Kidney Diseases.

19. Lucas GM, Lau B, Atta MG, Fine DM, Keruly J, et al. (2008) Chronic kidney disease incidence, and progression to end-stage renal disease, in HIV-infected individuals: a tale of two races. J Infect Dis 197: 1548-1557.

20. WHO (2007) Case Definitions of HIV for Surveillance and Revised Clinical Staging and Immunological Classification of HIV-related Disease in Adults and Children. Geneva: World Health Organization.

21. National Kidney Foundation (2002) K/DOQI clinical practice guidelines for chronic kidney disease: evaluation, classification, and stratification. Am J Kidney Dis 39: S1-266.

22. Andia I, Pepper LM, Mathieson P (2005) Prevalence of renal disease in patients attending the HIV/AIDS clinic at Mbarara University Teaching Hospital, Uganda ( Abstract TU Pe 15: 3 CO2).In: Book of Abstracts of 
Citation: Komolafe OO, Aderibigbe A, Olanrewaju TO, Chijioke A, Salami AK, et al. (2014) Microalbuminuria in a Cohort of Ambulatory HIVPositive Nigerians. J Nephrol Ther 4: 179. doi:10.4172/2161-0959.1000179

Page 6 of 6

the 3rd International AIDS society conference on HIV pathogenesis and treatment, Rio de Janeiro, Brazil.

23. Sumaili EK, Krzesinski JM, Zinga CV, Cohen EP, Delanaye P, et al. (2009) Prevalence of chronic kidney disease in Kinshasa: results of a pilot study from the Democratic Republic of Congo. Nephrol Dial Transplant 24: $117-122$.

24. Anthony S. Fauci, H (2005) Clifford Lane. Human Immunodeficiency Virus Disease: AIDS and Related Disorders, In: Harrisonâ $€^{\mathrm{m}} \mathrm{s}$ Principle of Internal Medicine, 16th Edition. Kasper DL, Braunwald E, Hauser SL et al (Eds), McGraw Hill, New York, pp 1073-1139.

25. Perinbasekar S, Brod-Miller C, Mattana J (1996) Absence of edema in HIV-infected patients with end-stage renal disease. J Acquir Immune Defic Syndr Hum Retrovirol 13: 368-373.

26. Erhabor O, Ejele OA, Nwauche CA, Buseri FI (2005) Some haematological parameters in human immunodeficiency virus (HIV) infected Africans: the Nigerian perspective. Niger J Med 14: 33-38.

27. Limone $\mathrm{P}$, Biglino A, Valle $\mathrm{M}$, , , et al. Insulin resistance in HIV-infected patients: relationship with pro-inflammatory cytokines. J Infect 2003 47: $52-58$.

28. Gould MM, Mohamed-Ali V, Goubet SA, Yudkin JS, Haines AP (1993) Microalbuminuria: associations with height and sex in non-diabetic subjects. BMJ 306: 240-242.
29. Cirillo M, Senigalliesi L, Laurenzi M, Alfieri R, Stamler J, et al. (1998) Microalbuminuria in nondiabetic adults: relation of blood pressure, body mass index, plasma cholesterol levels, and smoking: The Gubbio Population Study. Arch Intern Med 158: 1933-1939.

30. Bennett PH, Haffner S, Kasiske BL, Keane WF, Mogensen CE, et al. (1995) Screening and management of microalbuminuria in patients with diabetes mellitus: recommendations to the Scientific Advisory Board of the National Kidney Foundation from an ad hoc committee of the Council on Diabetes Mellitus of the National Kidney Foundation. Am J Kidney Dis 25: 107-112.

31. Emem CP, Arogundade F, Sanusi A, Adelusola K, Wokoma F, et al. (2008) Renal disease in HIV-seropositive patients in Nigeria: an assessment of prevalence, clinical features and risk factors. Nephrol Dial Transplant 23: 741-746.

32. Levey AS, Green T, Kusek JW, Rogers N, Roth D, et al. (1999) A more accurate method to estimate glomerular filtration rate from serum creatinine: a new prediction equation. Modification of Diet in Renal Disease Study Group. Ann Intern Med 130: 461-470.

33. Agaba EI, Wigwe CM, Agaba PA, Tzamaloukas AH (2009) Performance of the Cockcroft-Gault and MDRD equations in adult Nigerians with chronic kidney disease. Int Urol Nephrol 41: 635-642. 